Article

\title{
High Repetition Rate and Coherent Free-Electron Laser in the X-Rays Range Tailored for Linear Spectroscopy
}

\author{
Vittoria Petrillo ${ }^{1,2,3}$, Michele Opromolla ${ }^{1,2,3, *}$, Alberto Bacci ${ }^{2,3}$, Illya Drebot ${ }^{2,3}$, \\ Giacomo Ghiringhelli ${ }^{4}$, Alberto Petralia ${ }^{5}$, Ezio Puppin ${ }^{4}$, Marcello Rossetti Conti ${ }^{2,3}$ (D), \\ Andrea Renato Rossi ${ }^{2,3}$ (D) Alberto Tagliaferri ${ }^{4}$ (D), Sanae Samsam ${ }^{2,3,6}\left(\mathbb{D}\right.$ ) and Luca Serafini ${ }^{2,3}$ \\ and Giorgio Rossi ${ }^{1}$ \\ 1 Department of Physics, University of Milan, Via Celoria 16, 20133 Milano, Italy; \\ vittoria.petrillo@mi.infn.it (V.P.); giorgio.rossi2@unimi.it (G.R.) \\ 2 Istituto Nazionale di Fisica Nucleare-Sezione di Milano, Via Celoria 16, 20133 Milano, Italy; \\ alberto.bacci@mi.infn.it (A.B.); Illya.Drebot@mi.infn.it (I.D.); marcello.rossetti@mi.infn.it (M.R.C.); \\ andrea.rossi@mi.infn.it (A.R.R.); sanae.samsam@mi.infn.it (S.S.); luca.serafini@mi.infn.it (L.S.) \\ 3 Istituto Nazionale di Fisica Nucleare - Sezione di Milano, Laboratorio di Acceleratori e Superconduttivitá \\ Applicata, Via F.lli Cervi 201, 20090 Segrate, Italy \\ 4 Politecnico di Milano, Piazza Leonardo da Vinci, 32, 20133 Milano, Italy; \\ giacomo.ghiringhelli@polimi.it (G.G.); ezio.puppin@polimi.it (E.P.); alberto.tagliaferri@polimi.it (A.T.) \\ 5 European Nuclear Energy Agency, Via Enrico Fermi 40, 00044 Frascati, Italy; alberto.petralia@enea.it \\ 6 Department of Physics, Mohamed V University, 10000 Rabat, Marocco \\ * Correspondence: michele.opromolla@gmail.com or michele.opromolla@mi.infn.it
}

Received: 17 July 2019; Accepted: 3 September 2019; Published: 9 September 2019

\begin{abstract}
Fine time-resolved analysis of matter-i.e., spectroscopy and photon scattering-in the linear response regime requires fs-scale pulsed, high repetition rate, fully coherent $\mathrm{X}$-ray sources. A seeded Free Electron Laser (FEL) driven by a Linac based on Super Conducting cavities, generating $10^{8}-10^{10}$ coherent photons at $2-5 \mathrm{keV}$ with $0.2-1 \mathrm{MHz}$ of repetition rate, can address this need. Three different seeding schemes, reaching the X-ray range, are described hereafter. The first two are multi-stage cascades upshifting the radiation frequency by a factor of 10-30 starting from a seed represented by a coherent flash of extreme ultraviolet light. This radiation can be provided either by the High Harmonic Generation of an optical laser or by an FEL Oscillator operating at 12-14 nm. The third scheme is a regenerative amplifier working with X-ray mirrors. The whole chain of the $\mathrm{X}$-ray generation is here described by means of start-to-end simulations.
\end{abstract}

Keywords: FEL seeding; compact FEL

\section{Introduction}

Fine analyses of matter, based on techniques such as ultrafast coherent-X-ray spectroscopy, inelastic photon scattering and with applications extending from the life sciences to material physics, are currently performed with two main types of X-ray sources: synchrotron radiation (SR) sources and Free Electron Lasers (FELs) driven by linear electron accelerators (Linacs) in the self-amplified spontaneous emission (SASE) mode. Pioneering FEL sources [1-3], new X-ray FELs [4-7] and novel constructions or projects underway worldwide [8-10] provide or foresee ultra-bright pulses of 10-100 fs duration, with time and intensity jitter from pulse to pulse determined by the intrinsic fluctuations of the SASE process. So far FERMI@Elettra [11,12] is the only fully coherent FEL in operation, amplifying a Ti:Sa seed pulse with negligible time jitter and minor pulse-to-pulse intensity fluctuations; it operates 
down to wavelengths of $4 \mathrm{~nm}$ and at a repetition rate up to $50 \mathrm{~Hz}$. In general, the FEL sources provide extremely brilliant pulses with more than $10^{12}$ photons per pulse of $<100 \mathrm{fs}$ duration. Much of the success of FEL-based experiments, which are often compatible with single pulse detection, has been in the study of the internal structure or ordering of materials (solids, molecules, atoms). On the contrary, spectroscopic probes, suitable for the study of magnetic and electronic structures, are more demanding in terms of average photon flux on the sample and are thus harder to implement at low repetition rates. Time resolution of 10-100 fs has emerged as a need of fine analysis also in spectroscopy, that, on the other hand, requires short pulses and a contained photon number per shot so that the spectral density remains instantaneously within the linear response limit. Pump probe photoemission experiments of high scientific impact in atomic, molecular, nanoparticle and solid state physics require indeed a source of ultra-short photon pulses (order $10 \mathrm{fs}$ ) with about $10^{8}$ photons/pulse to remain, according to photoionization cross sections, in the linear or near-linear response regime that allows spectroscopy to be interpreted within perturbative approaches, and repetition rates as high as possible ( $\mathrm{MHz}$ range) for collecting adequate statistics in short times.

Currently available FEL sources are quite far from being ideal for spectroscopy, as the number of photons per pulse exceeds by $2-4$ orders of magnitude the one compatible with the linear response regime: severe attenuation of the pulses is therefore required for photoemission or X-ray absorption spectroscopy (XAS) and X-ray Magnetic circular dichroism (XMCD) experiments and this wastes an astounding fraction of the FEL operating energy. The warm Linacs are limited to a few tens of $\mathrm{Hz}$ repetition rate, up to $100 / 120 \mathrm{~Hz}$, which is definitely non ideal for collecting adequate statistics in high resolution spectroscopy. The signal from the EU-XFEL, shaped in 2700 micropulses at $4.5 \mathrm{MHz}$ in 10 macropulses per second, is also not ideal for spectroscopy-from one hand attenuation is needed and from the other one the repetition rate of the micropulses is so high that it overruns the present capabilities of detectors and of possible pump-probe set-up operation. Severe limitations to spectroscopy with X-ray FELs are set by the phase and intensity fluctuations typical of the SASE mode.

There is therefore the clear scientific need for a novel type of source that would provide $10 \mathrm{fs}$ coherent pulses of $10^{8}$ photons at $0.5-2 \mathrm{MHz}$, able to bridge between the most advanced SR and the current FEL sources. Full seeding, successfully done at FERMI in the XUV-soft X-rays range, should be ideally extended to X-ray energies.

These demanding requests about pulse structure, intensity, repetition rate, reduced jitter and true coherence, able to cover the range in time resolution and average photon flux between SR and current FELs, are addressed by conceiving a tailored source based on a seeded FEL driven by a Linac based on Super Conducting cavities, providing $10^{8}-10^{10}$ coherent photons at $2-5 \mathrm{keV}$ and at least $500 \mathrm{kHz}$ of repetition rate.

The production of coherent radiation in the $\mathrm{X}$-ray spectral range is a very demanding research field. Laser's High Harmonic Generation provide sources of coherent radiation up to $1-2 \mathrm{keV}$ of photon energy, but with intensity many orders of magnitude lower than FELs. A partial longitudinal coherence can be obtained by following the road of the single spike SASE [13] regime or by means of self-seeding processes [14]. If full coherence is needed, a seeded amplifier has to be implemented, where the radiation does not start from the electron shot noise, but is forced by an external coherent source, which enables to reach a higher degree of temporal coherence within a shorter distance. However, the direct seeding [15] is not possible in the soft-hard X-rays range due to the lack of a coherent seed at such short wavelengths. Other seeding schemes should be therefore analyzed. HGHG multistage cascades seeded with harmonics of an IR laser generated in crystals, shown at SPARC in the optical-UV range [16], then demonstrated and currently used at FERMI up to few nm of wavelength [12], are difficult to implement in the hard X-rays range. The technique of the EEHG [17-20] is actually successfully demonstrated up to the nanometric range of wavelengh. Its implementation in the Tender/Hard X rays regime is however not guaranteed, due to phase errors in the seed laser and e-beam instabilities. FEL oscillators or regenerative amplifiers [21] have been proposed as direct source of X-rays (XFELO) [22] or as seeding source for a subsequent cascade [23], but the operational 
scenario proposed so far, with electrons at several GeVs, leads to the necessity for extremely long and expensive accelerator devices, setting strict limits on this option.

The electron beam we will use in the following is supposed to be generated by the accelerator of the MariX project (Multi-disciplinary Advanced Research Infrastructure for the generation and application of X-rays) [24], which should provide 2-3.8 GeV electron beams with an emittance of $0.3 \mathrm{~mm}$ mrad and at few $10^{-4}$ of energy spread. The FEL source MariX here described is a compact infrastructure, suitable to be constructed in medium size research centers and university campuses. It is specifically tailored for time-resolved spectroscopic applications with coherent $X$-rays, where the individual pulses should not exceed the linear response regime and space charge effects. This implies $10^{7}-10^{10}$ photons at the exit of the FEL per $10 \mathrm{fs}$-long pulses at several hundred $\mathrm{kHz}$ of repetition rate. These pulses are at most $3-4$ orders of magnitude lower than the individual peak intensity of the current X-FELs. The 4-5 orders of magnitude gain in repetition rate allowed by MariX, for example, at $0.5-2 \mathrm{MHz}$, restores the high flux per second of the most advanced synchrotron sources, whilst having ultrashort pulses suitable for time resolved pump-probe methods in optical, photoelectric effect and inelastic $X$ scattering experiments. With the current FEL technology providing $10^{11}-10^{13}$ photons per shot, instead, a $10^{3}-10^{5}$ attenuation of $X$-ray beams must be applied in order to measure an undistorted, non energy shifted core level spectrum, while the $30-50 \mathrm{~Hz}$ operation limits the statistics of these measurements to a $10^{5}$ lower time integrated flux per second with respect to MariX.

High longitudinal coherence will enable pump-probe methods at 10-100 fs accuracy and with high statistics. This option will actually benefit precision timing measurements as the SASE intrinsic pulse-to-pulse jitter will be substantially eliminated, as well as the reduced pulse-to-pulse intensity fluctuations, thus approaching, at X-ray energies, the unique performance of FERMI@Elettra [12].

In this paper, we show a comparison between three different methods for producing truly coherent X-ray pulses specifically conceived for the MariX project: (i) a harmonic cascade seeded by the harmonics of an IR laser, generated in gas; (ii) a similar cascade, but seeded by an ultraviolet FEL oscillator driven by the same electron beam alimenting the FEL cascades and (iii) a regenerative amplifier in the X-rays. The first option is based on a seed provided by the High Harmonic Generation in gas of an optical laser light. In order to obtain Ångström-class radiation, the seed at 12-14 nm is injected into a triple cascade with an up-shift frequency factor of 10-30. In the second case, the electron beam exiting the Linac at an energy of about $3 \mathrm{GeV}$ permits the oscillator to radiate at about $13 \mathrm{~nm}$, a wavelength value for which efficient multilayer mirrors exist. The $\mathrm{n}$-th harmonics of the fundamental of the oscillator radiation is used as a seed for a double cascade. Regarding the third option, diamond mirrors and beam splitters working at $3 \mathrm{keV}$ energy should allow the synchronization of the radiation pulses with the electrons. In all cases, an electron beam characterized by relatively low energy $(2-2.5 \mathrm{GeV})$ has been used, generated by a relatively short accelerator of compact footprint; also the use of short period undulators and the choice of the seeding methods follow the trend of containing infrastructure costs and dimensions.

Hereafter, we will discuss nominal parameters and start to end simulations of each of the three cases. Final comments will be presented in the conclusions.

\section{FEL Layout and Start-To-End Simulations}

Among all the methods for producing coherent X-ray radiation, here we analyze the cascaded harmonic generation alimented by a seed obtained with HHG in gases (Section 2.2) and by the radiation produced by a FEL oscillator (Section 2.3). The third case (Section 2.4) is instead an X-ray regenerative amplifier.

All our analyses rely on an electron beam with a Gaussian longitudinal current profile, having the same properties of the electron beam foreseen by the accelerator of the MariX project [24]. MariX is based on the innovative design of a two-pass two-way superconducting linear electron accelerator, equipped with an arc compressor [25-27], to be operated in CW mode at $1 \mathrm{MHz}$. The characteristics of the electron beam are listed in Table 1 [28]. 
Table 1. Electron beam for MariX Free Electron Laser (FEL).

\begin{tabular}{cccccc}
\hline Electron Beam Energy & $\mathrm{GeV}$ & $\mathbf{1 . 6}-\mathbf{3 . 8}$ & rms Normalized Emittance & $\mathbf{m m}$ mrad & $\mathbf{0 . 3 - 0 . 5}$ \\
\hline Charge & pC & $8-50$ & rms relative energy spread & $10^{-4}$ & $2-4$ \\
Current & kA & $1.3-1.6$ & electron beam duration & fs & $2.5-16$ \\
\hline
\end{tabular}

After the second passage through the Linac, the electron beam is matched to the undulator device. In order to obtain radiation in the desired wavelength range (5-2 A, or in energy unit 2-5 keV) with the moderate electron energy provided by the MariX accelerator (at maximum $3.8 \mathrm{GeV}$ ), a short period undulator must be considered. It follows from the resonance relation that a period of $\lambda_{w}=1.2 \mathrm{~cm}$ is suitable. The structure of the undulator, instead, depends on the seeding scheme. HGHG cascades need segmented undulators, while for a regenerative amplifier configuration a single module of short pass undulator, equipped with a hard X-ray sequence of mirrors, is sufficient.

\subsection{SASE Mode}

Considering the electron beam parameters of Table 1 , and a not segmented undulator of period $\lambda_{w}=1.2 \mathrm{~cm}$ and strength $a_{w}=0.65$, the SASE radiation obtained at about $4 \AA$ is shown in Figure 1 , left panel: case (a) refers to high charge multispike SASE regime; case (b) to low charge single spike regime. The coherence degree, evaluated as

$$
\Gamma_{1,2}(t)=\frac{\left|\int d t^{\prime} E_{1}\left(t^{\prime}\right) E_{2}\left(t-t^{\prime}\right)\right|}{\sqrt{\int d t^{\prime}\left|E_{1}\right|^{2} \int d t^{\prime}\left|E_{2}\right|^{2}}}
$$

is shown in the lateral boxes of Figure 1 in the cases of one shot $\Gamma=\Gamma_{1,1}$ and between two uncorrelated shots. In this expression, $E_{1,2}$ is the complex electric field of two pulses as function of time $t$.
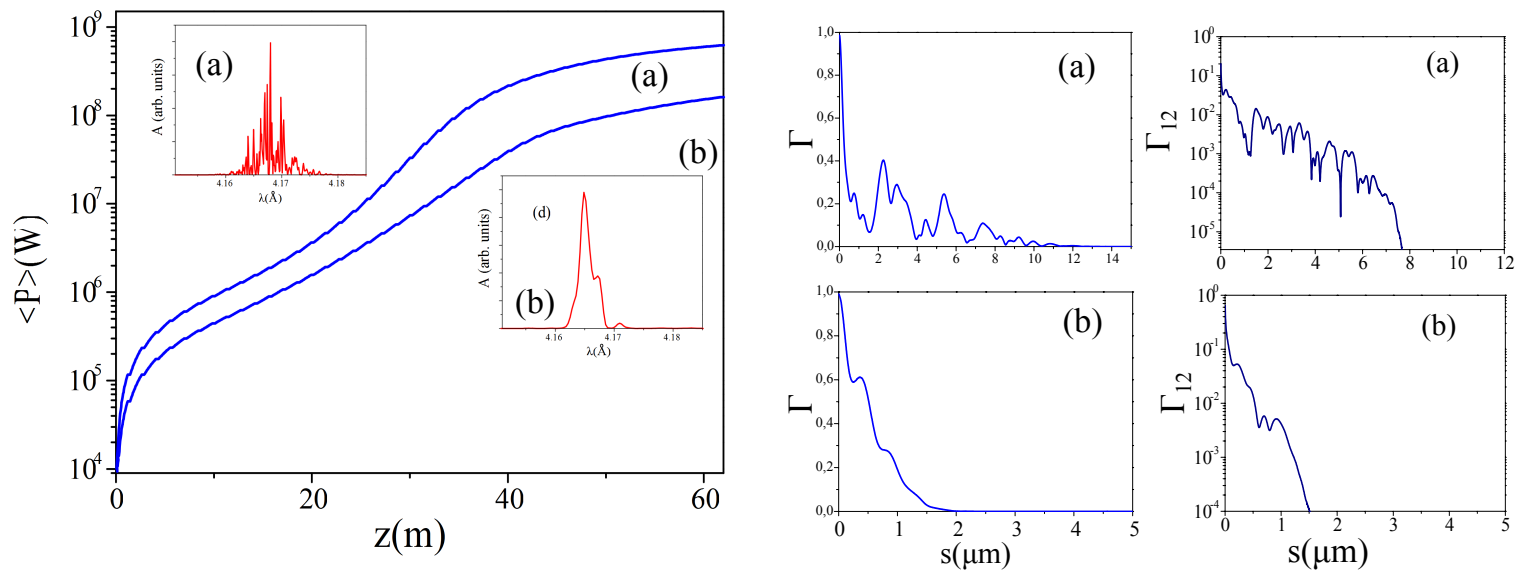

Figure 1. SASE radiation at $4.16 \AA$. Case (a) refers to high charge multispike self amplified spontaneous emission (SASE) regime, case (b) to low charge single spike regime. (Left panel): radiation growth vs. undulator coordinate; (Right panels): one shot coherence degree $\Gamma$ and mutual coherence degree between two uncorrelated shots $\Gamma_{12}$ as function of the longitudinal coordinate.

\subsection{Harmonic Cascade Seeded by the Harmonics of an IR Laser Generated in Gas}

The seeding technique based on High Harmonic Generation (HHG) permits to produce radiation with the same energy level as the SASE mode, but with full temporal coherence and small energy fluctuations. In this configuration, external laser pulses, or their harmonics, are injected as seed into a first undulator segment, where they induce the electron beam to radiate the laser frequency itself and its harmonics. A further undulator segment - the radiator-is tuned to the n-th harmonics of the radiation of the previous stage, so that the electron beam is forced to emit coherently at this 
resonant frequency. The temporal and spectral distribution of the output radiation are determined by the seed laser, that encodes its coherence degree on the FEL radiation. Seeded FEL amplifier operation in combination with harmonic generation has been demonstrated experimentally at both mid-infrared and VUV wavelengths $[12,16,29]$. The implementation of this scheme in the hard X-ray spectral range, however, has still to be proven. The most suitable sources for this scope are the Ti:Sa lasers, thanks to their characteristics in terms of energy (J-class) and pulse shortness (fs-class). Besides reproducibility and total pulse energy, they have to guarantee stability, mode quality and the possibility of a precise matching of the radiation into the first modulator. These lasers, however, do not provide intense radiation below $120 \mathrm{~nm}$ of wavelength. A solution that can be explored is a seeding setup based on High-order Harmonic generation in Gas (HHG) [30-32], that represents one of the most promising methods for generating ultrashort pulses of coherent radiation in the XUV (30-300 eV)-soft X (300-3000 eV) region of the spectrum, suitable to seed short wavelength Free Electron Lasers. High-Order harmonics of an ultra-short laser pulse show high temporal and spatial coherence and are characterized by a time duration shorter than that of the driving source; XUV pulses with durations ranging from 8 to 13 femtoseconds have been generated by spectral selection of a single harmonic with a suitable time-compensated monochromator. HHG sources are inherently inefficient: for this reason, exploiting HHG for FEL seeding requires powerful laser sources and suitable techniques to boost the XUV emission to the required peak powers.

Though substantial energy from this type of source is available in the VUV, only few experiments have been performed so far with HHG sources as seeding for FEL [33] and no one in the EUV-soft-hard $X$-rays range. From different studies starting from a Ti:Sa laser at $800 \mathrm{~nm}$, it emerges the possibility of having radiation on the 58th-65th harmonics corresponding to wavelengths between 14 and $12 \mathrm{~nm}$. Approaching this range, however, a high seed energy is required to overcome the electron shot noise level, that increases rapidly with decreasing wavelength. The energy generated in all schemes is around 3-15 nJ and the time lengths of the single radiation pulse is tens of femtoseconds. These values, if reproducible, could make a seeding scheme with HHG on MariX feasible.

The cascade considered for MariX is shown in Figure 2 and consists of two stages of longer period modulators and a third chain of short period undulator as radiator. The two modulators are respectively of period $\lambda_{w}=5 \mathrm{~cm}$ and $2.8 \mathrm{~cm}$ and lengths $10 \mathrm{~m}$ and $15 \mathrm{~m}$. To be resonant at $\lambda=13.6 \mathrm{~nm}$, the resultant undulator parameter of the first modulator is $a_{w}=3.12$, fully compatible with its specifics. The second modulator operates at $a_{w}=1.68$. The radiator consists of undulators with a period of $1.2 \mathrm{~cm}$ and $a_{w}=0.89$ to resonate at about $0.544 \mathrm{~nm}$, corresponding to the 5 th harmonics of the second modulator. The fresh bunch technique [34] has been used, by superimposing the seed on a part of the electron beam not deteriorated by the radiation process in the previous stage.

A summary of the undulators' parameters is shown in Table 2. A pulse with a wavelength of $13.6 \mathrm{~nm}$, an energy of 10-15 nJ and a duration of $30 \mathrm{fs}(\mathrm{rms})$ has been chosen as reference seed for the simulations of the FEL cascade.

Table 2. Undulator parameters and radiation wavelengths for the High Harmonic Generation (HHG) seeded cascade. Electron beam energy = 2.2 GeV.

\begin{tabular}{cccccc}
\hline & $\lambda_{w}$ & $\boldsymbol{a}_{w}$ & Fund & 3rd Harm & 5th Harm \\
\hline 1st Modulator & $5 \mathrm{~cm}$ & 3.12 & $13.6 \mathrm{~nm}$ & $4.53 \mathrm{~nm}$ & $2.72 \mathrm{~nm}$ \\
2nd Modulator & $2.8 \mathrm{~cm}$ & 1.68 & $2.72 \mathrm{~nm}$ & $0.906 \mathrm{~nm}$ & $0.544 \mathrm{~nm}$ \\
Radiator & $1.2 \mathrm{~cm}$ & 0.89 & $0.544 \mathrm{~nm}$ & & \\
\hline
\end{tabular}

The results obtained, starting from $13.6 \mathrm{~nm}$ and up-shifting the frequency by a factor $5 \times 5$, are presented in Figure 3. The final yield is of $10 \mu \mathrm{J}$ of radiation corresponding to approximately $10^{10}$ photons per shot and $10^{15}$ photons $/ \mathrm{s}$. The characteristics of the radiation after $40 \mathrm{~m}$ of undulator (after $10 \mathrm{~m}$ of the radiator) are summarized in Table 3. 


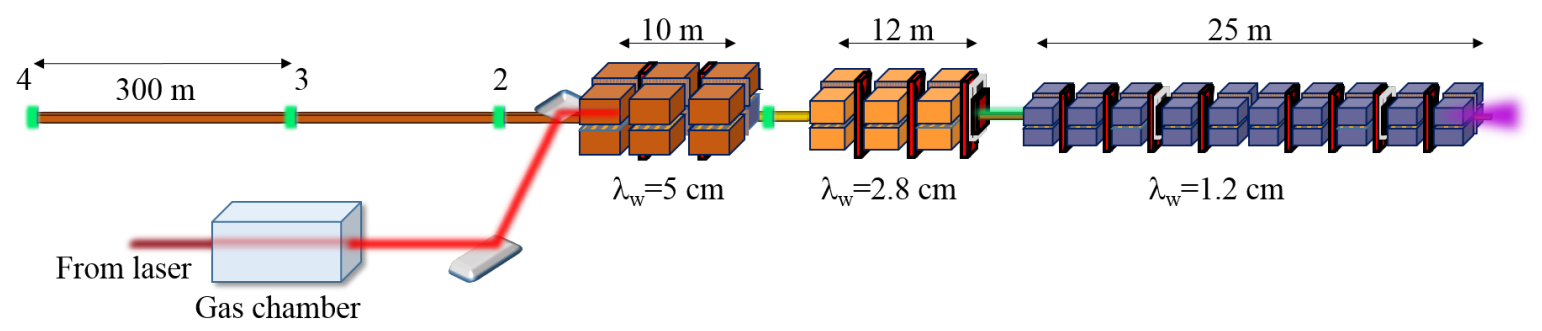

Figure 2. Segmented undulator scheme for a three-stage cascade with HHG as seed.

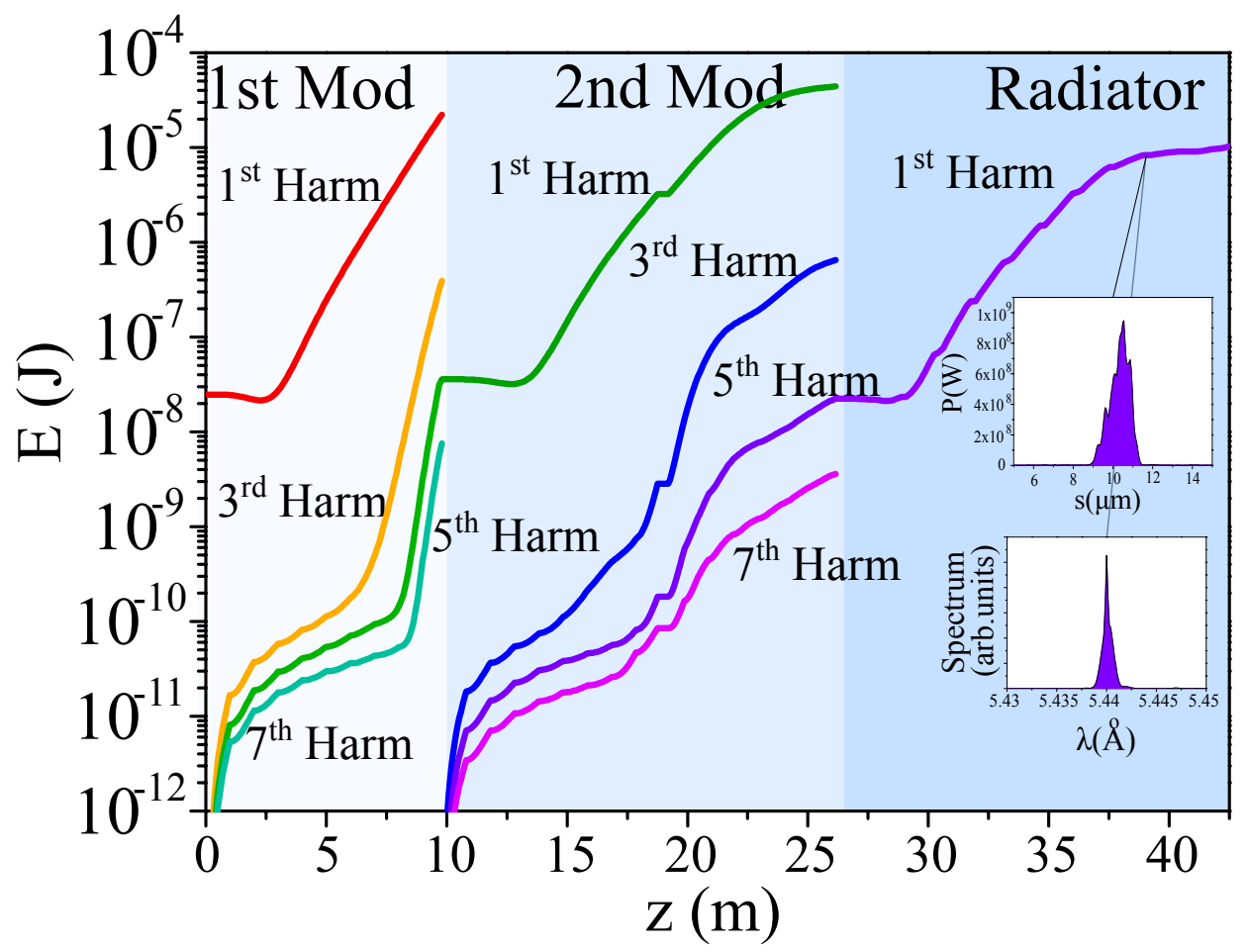

Figure 3. $5 \times 5$ cascade seeded by HHG: FEL radiation energy vs undulator position, temporal power distribution and spectrum (in the boxes) extracted at about $40 \mathrm{~m}$. Seeded cascaded fresh-bunch case. First modulator (1st Mod) with $5 \mathrm{~cm}$ period. Second modulator ( $2 \mathrm{nd} \mathrm{Mod}$ ) with $2.8 \mathrm{~cm}$ period. Radiator with $1.2 \mathrm{~cm}$ period. The FEL simulations have been performed with GENESIS 1.3 [35].

Table 3. Radiation characteristics of the HHG seeded cascade. $\lambda$ is the radiation wavelength, $E$ the radiation energy, $\mathrm{N}$ /shot the numberof photons per shot, $\mathrm{N} / \mathrm{s}$ the average number of photons, bw the relative r.m.s. bandwidth, div means divergence, length and size are the longitudinal and transverse rms pulse dimensions. The repetition rate of the source is assumed to be $100 \mathrm{kHz}$.

\begin{tabular}{cccc}
\hline$\lambda$ & $\mathbf{0 . 5 4 4} \mathbf{n m}$ & $\mathrm{E}$ & $\mathbf{1 0} \boldsymbol{\mu J}$ \\
\hline $\mathrm{N} /$ shot & $10^{10}$ & $\mathrm{~N} / \mathrm{s}$ & $10^{15}$ \\
bw & $0.07 \%$ & length & $2-3 \mu \mathrm{m}$ \\
div & $35 \mu \mathrm{rad}$ & size & $75 \mu \mathrm{m}$ \\
\hline
\end{tabular}

All seeding schemes based on laser harmonics are difficult to implement at repetition rates larger than $100 \mathrm{kHz}$, due to the insufficient laser pulse energy (under the shot noise level) at increasing repetition rates, impeding the seeding process. The techniques described in the following sections overcome this limitation. 


\subsection{Harmonic Cascade Seeded by an FEL Oscillator}

In this second option, shown in Figure 4, the seed is delivered by an oscillator working at $\lambda=13.6 \mathrm{~nm}$. Since the electron beam alimenting the system turns out to be deteriorated by the radiation process, it is dumped after each passage into the oscillator undulator module. Therefore, the $1 \mathrm{MHz}$ electron beam trains are alternatively driven into the oscillator and into the FEL cascade, with a resulting repetition rate of $0.5 \mathrm{MHz}$.

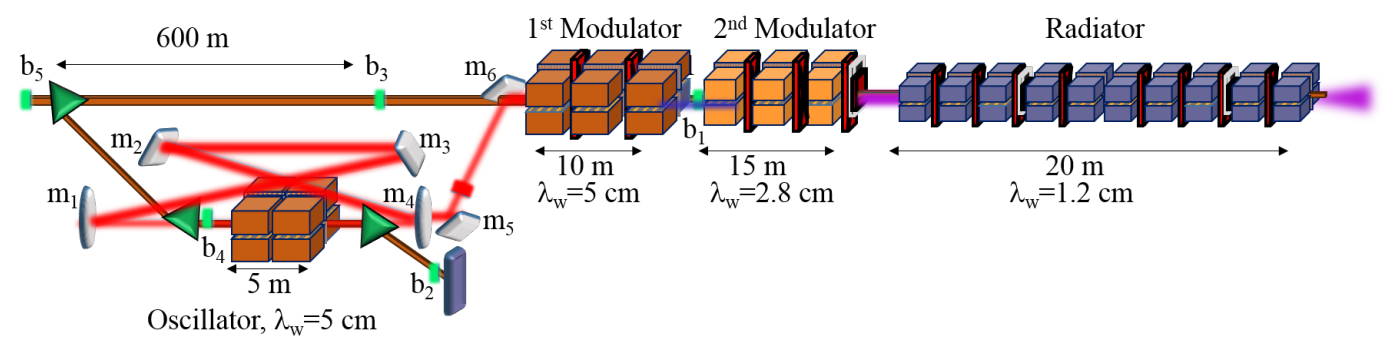

Figure 4. Segmented undulator scheme for a three-stage cascade driven by an Extreme Ultraviolet-FEL Oscillator.

The oscillator is constituted by a $6 \mathrm{~m}$ long undulator segment with period $\lambda_{w}=5 \mathrm{~cm}$ and $a_{w}=2.08$. It emits at $13.6 \mathrm{~nm}$, corresponding to the optimum wavelength for Mo/Si multilayer mirrors [36]. The reflectivity of the mirrors exceeds $\mathrm{R}=0.75$ at normal or quasi-normal incidence, with the width of the optical line transfer function much larger than the SASE or seeded radiation spectra, so the spectral filtering of the mirrors is in this case inessential. The purification of spectral and temporal distributions occurs instead via reiterated amplification of the best SASE spike. The reflectivity after four reflections is $\mathrm{R}=0.3$, and the fraction coupled to the first modulator is about $25 \%$ of the oscillator yield. The oscillator saturates in 100 passages and the modest oscillations around the average values of intensity and frequency are due to the different microscopy of the electron beams.

The simulation of the oscillator is shown in Figure 5, which shows the intracavity energy growth as function of the number of cycles, together with the final spectral and temporal densities taking into account the mirror, the optical line and the slippage effects. The characteristics of the seed are summarized in Table 4. Since the seed radiation exiting the cavity should meet the successive electron beam at the entrance of the modulator, it has to be retarded by $1 \mu \mathrm{s}$, corresponding to a total length of about $300 \mathrm{~m}$. Other $n(n=1, \ldots 3)$ couples of mirrors are foreseen in this section of the path, with a total attenuation of $R^{2 n}$.

The seed is then synchronized and superimposed to the electron beam at the entrance of the first modulator. The results of a cascade performing a $5 \times 5$ frequency upshift, starting from a FEL oscillator seed, are shown in Figure 6 and summarized in Table 5.

Table 4. Characteristics of the seed generated by the FEL oscillator at the entrance of the first modulator. Symbols are explained in Table 3.

\begin{tabular}{cccc}
\hline$\lambda$ & $\mathbf{1 3 . 6} \mathbf{~ n m}$ & $\mathbf{E}$ & $\mathbf{5 0 ~} \mathbf{~ J}$ \\
\hline $\mathrm{N} /$ shot & $3.4 \times 10^{9}$ & $\mathrm{~N} / \mathrm{s}$ & $1.7 \times 10^{15}$ \\
$\mathrm{bw}$ & $0.15 \%$ & rms length & $2 \mu \mathrm{m}$ \\
div & $85 \mu \mathrm{m}$ & size & $100 \mu \mathrm{m}$ \\
\hline
\end{tabular}


Table 5. Characteristics of the radiation of a $5 \times 5$ cascade seeded by a FEL oscillator. Symbols are explained in Table 3.

\begin{tabular}{cccc}
\hline$\lambda$ & $\mathbf{5 . 4 4} \AA$ & $\mathbf{E}$ & $\mathbf{1 1} \boldsymbol{\mu} \mathbf{J}$ \\
\hline $\mathrm{N} /$ shot & $3 \times 10^{10}$ & N/s & $1.5 \times 10^{16}$ \\
bw & $0.017 \%$ & rms length & $2 \mu \mathrm{m}$ \\
div & $3.6 \mu \mathrm{m}$ & size & $24 \mu \mathrm{m}$ \\
\hline
\end{tabular}

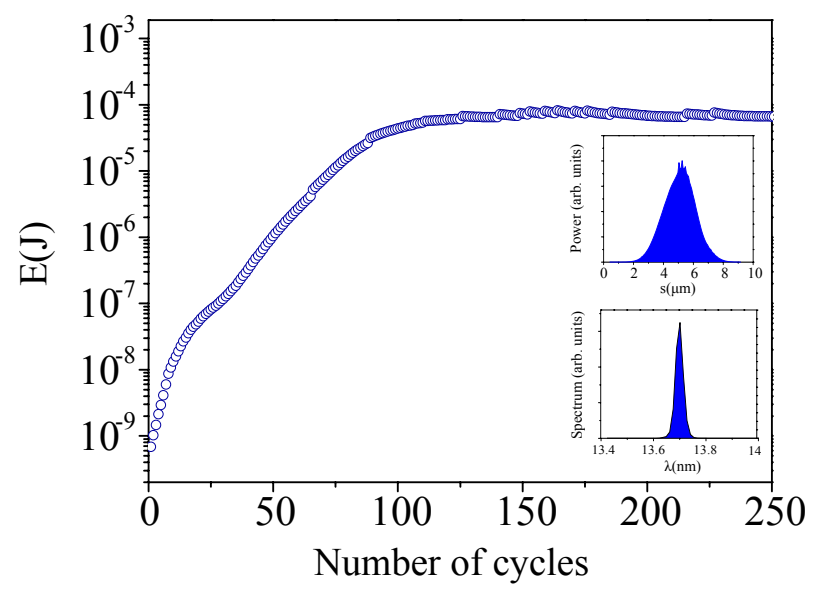

Figure 5. Intracavity energy vs number of cycles. In the boxes: radiation amplitude and spectrum at saturation.

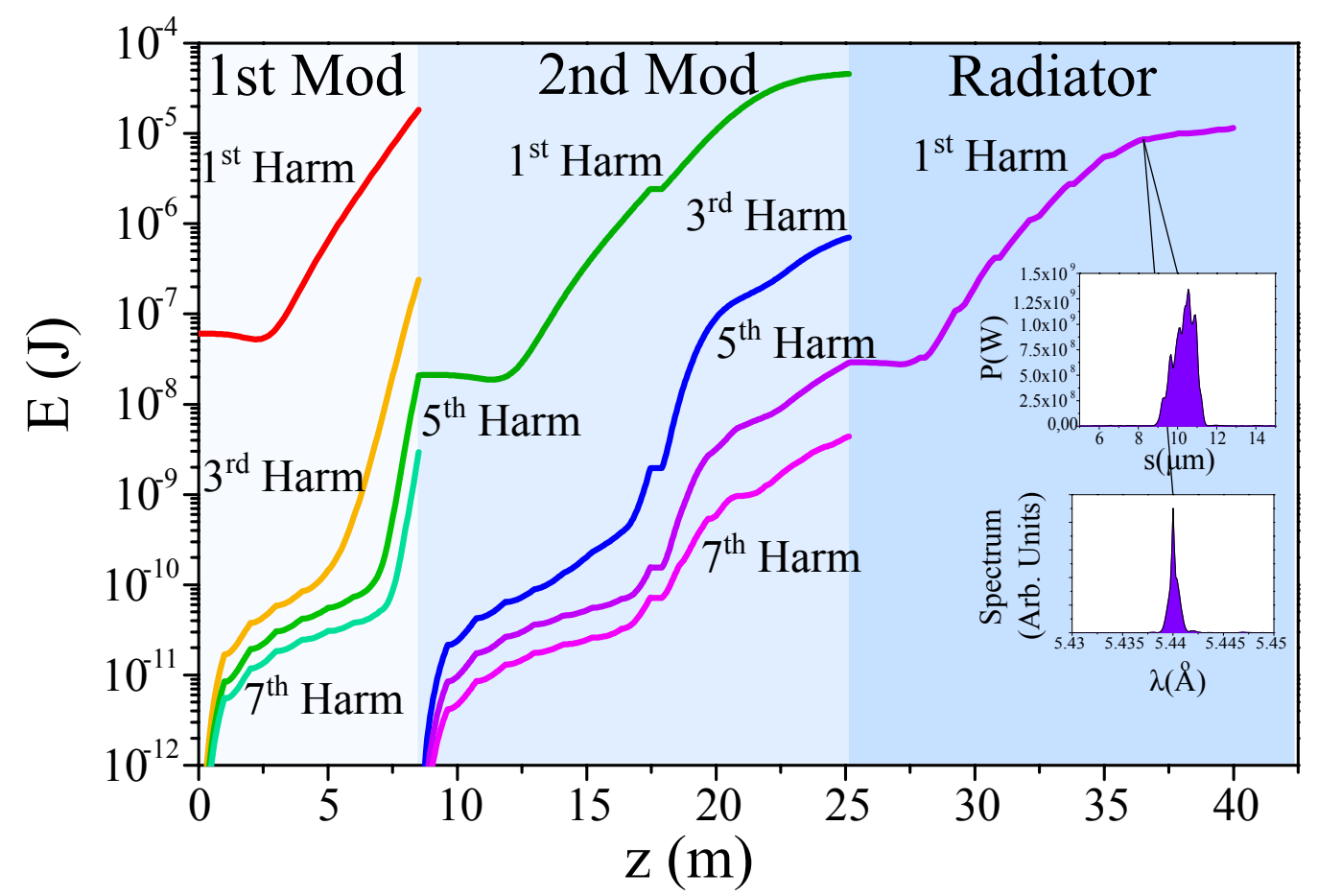

Figure 6. $5 \times 5$ cascade with the seed provided by a FEL oscillator: FEL energy of fundamental and harmonics generated in the various modules are presented vs the coordinate $\mathrm{z}$ in the undulator. Seeded cascaded fresh-bunch case. First modulator (1st Mod) with $5 \mathrm{~cm}$ period. Second modulator (2nd Mod) with $2.8 \mathrm{~cm}$ period. Radiator with $1.2 \mathrm{~cm}$ period. The FEL simulations have been performed with GENESIS 1.3 [35]. In the boxes, the temporal and spectral distrubution of the pulse. 


\subsection{Regenerative Amplifier}

The third option analyzed is a regenerative amplifier (see Figure 7) where the sequence of the beam packets entering the undulator is synchronized with the radiation reflected and recirculated by hard X-rays mirrors and a beam splitter. The cavity contains three diamond mirrors and one beam splitter, operating at $4.17 \AA$. Figure 8 presents the transfer function $\mathrm{T}$ of the optical line as a function of the photon energy at the chosen frequency for quasi-orthogonal reflection. In this case, the transfer function is much narrower than the natural FEL spectral line and the spectral filtering of the mirrors is the dominant effect in the spectral width reduction. The action of the mirrors at the n-th passage is evaluated by computing the convolution between the electric field at the exit of the undulator and the Fourier anti-transform $\widetilde{T}$ of the transfer function:

$$
E_{n+1}^{\text {in }}(t)=\frac{1}{\sqrt{2 \pi}} \int d t^{\prime} \widetilde{T}\left(t-t^{\prime}\right) E_{n}^{\text {out }}\left(t^{\prime}\right)
$$

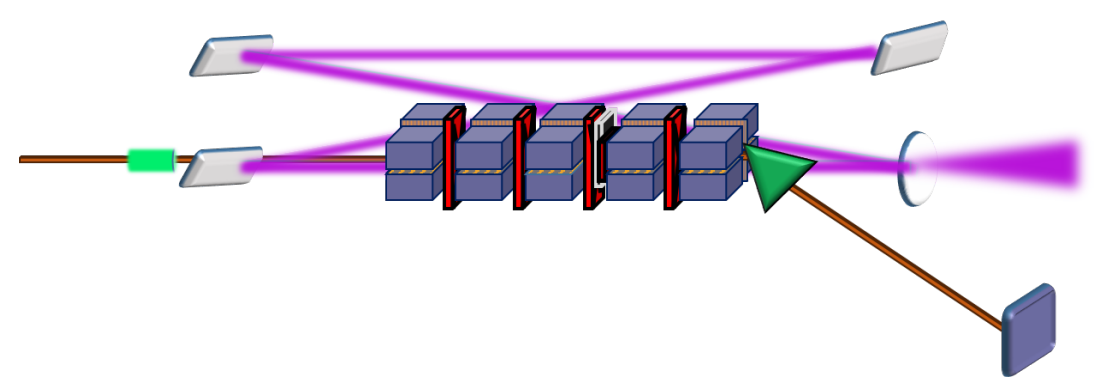

Figure 7. Regenerative Amplifier with three diamond mirrors and one beam splitter operating at different wavelengths.

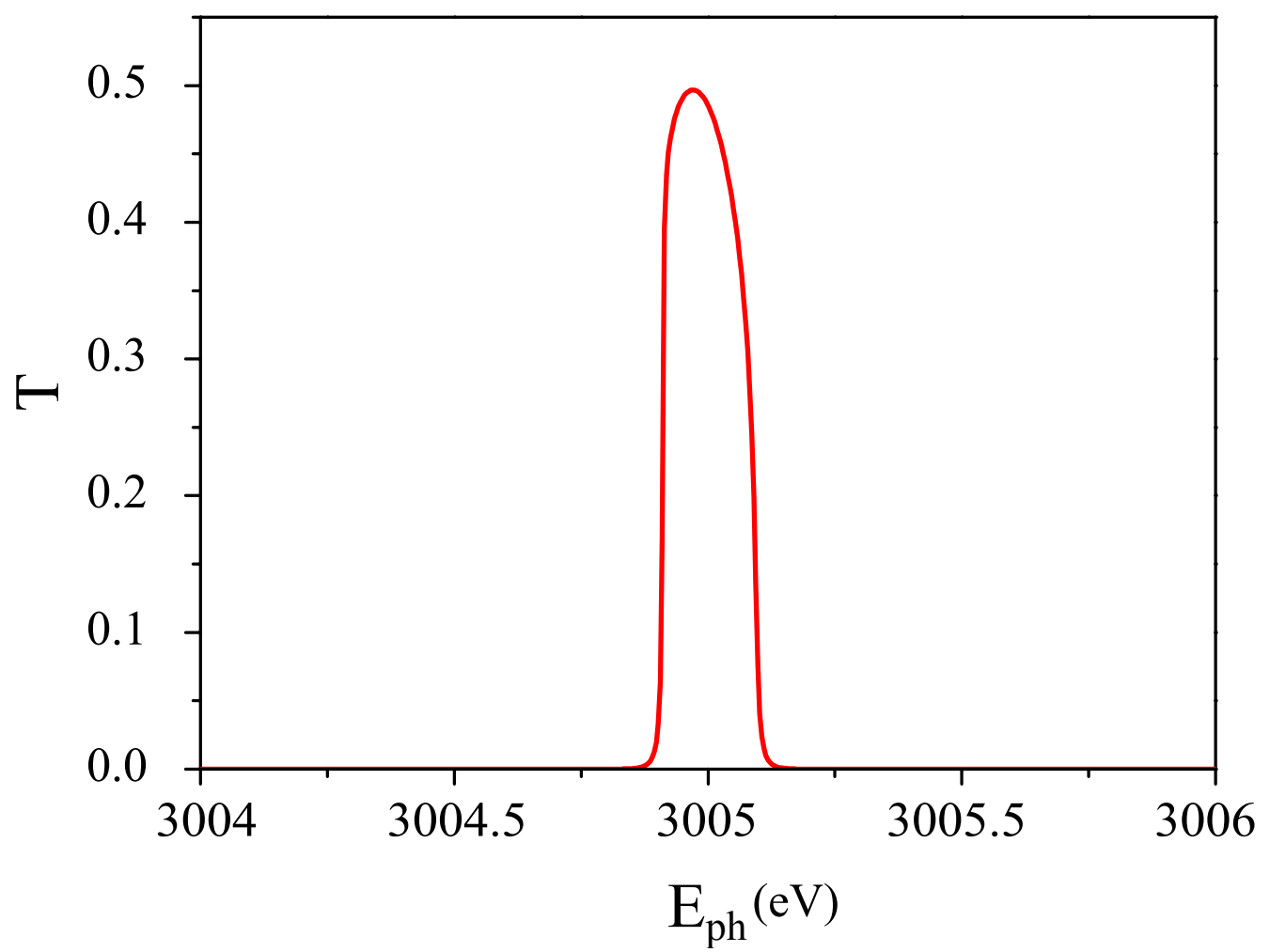

Figure 8. Transfer function of an optical line made by three diamond mirrors and one beam splitter vs. the photon energy. 
Figure 9 presents the growth of the intracavity energy as function of the round trip number. The radiation characteristics are listed in Table 6 . The operational details of the cavity are the object of current investigations and will be presented in future works.

Table 6. Radiation characteristics of the regenerative amplifier. The repetition rate of the source is $1 \mathrm{MHz}$. Symbols are explained in Table 3 .

\begin{tabular}{cccc}
\hline$\lambda$ & $4.16 \AA$ & $\mathrm{E}$ & $21 \mu \mathrm{J}$ \\
\hline $\mathrm{N} /$ shot & $4.4 \times 10^{10}$ & $\mathrm{~N} / \mathrm{s}$ & $4.4 \times 10^{16}$ \\
$\mathrm{bw}$ & $0.4 \%$ & length & $5 \mu \mathrm{m}$ \\
$\mathrm{div}$ & $14 \mu \mathrm{rad}$ & size & $38 \mu \mathrm{m}$ \\
\hline
\end{tabular}

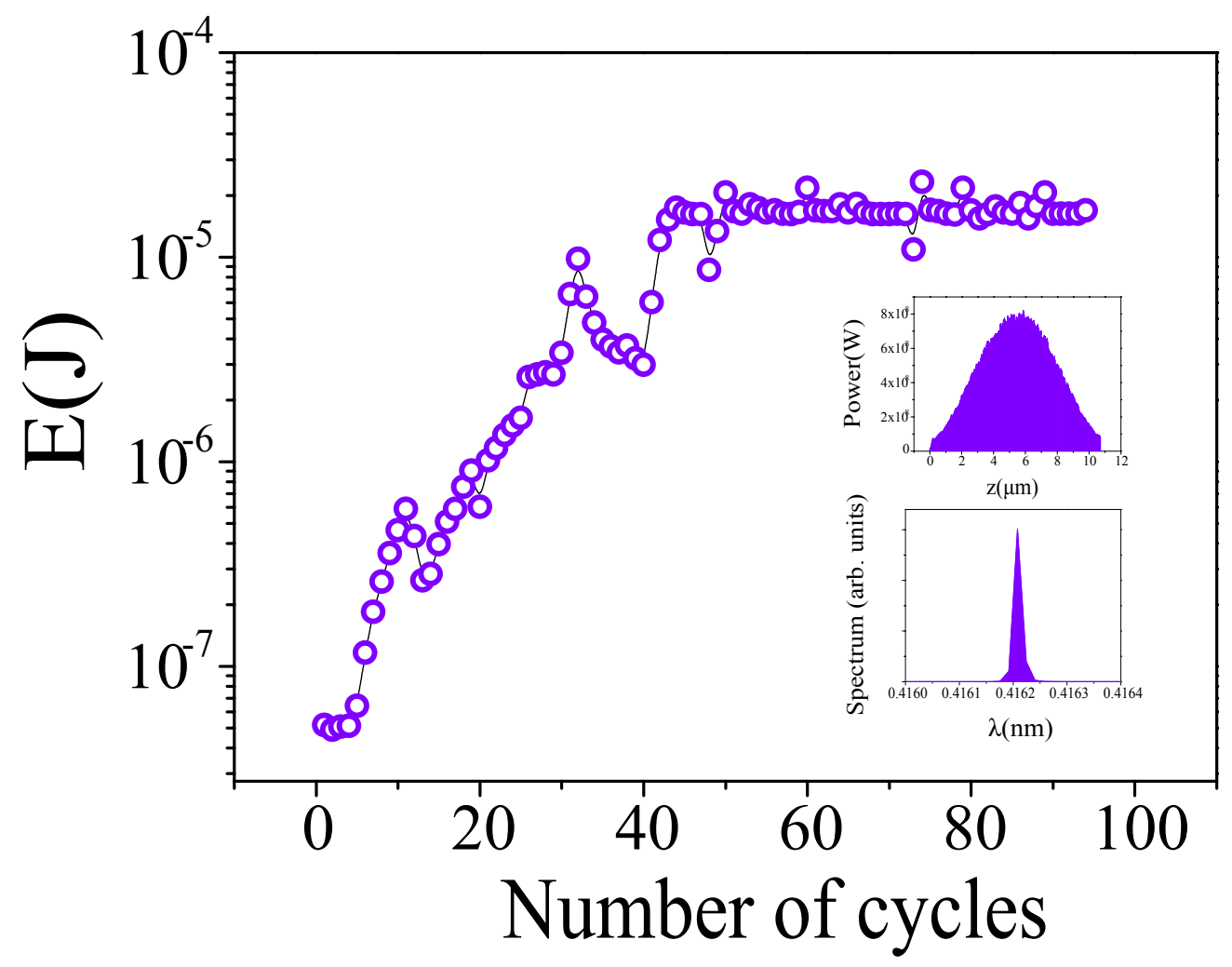

Figure 9. Intracavity radiation of the regenerative amplifier at $4.16 \AA$. In the boxes: power at saturation and spectrum.

\section{Conclusions}

In the Angstrom wavelength range, MariX provides either $10^{10}-10^{11}$ photons per shot with a repetition rate of $1 \mathrm{MHz}$ in SASE mode or $10^{9}-10^{10}$ photons per shot in the single spike SASE mode. These estimations, that do not take into account degradations due to errors, misalignments and jitters, exceed by one or more orders of magnitude the target values set by the scientific case. MariX will be therefore capable to satisfy the requested FEL photon beam parameters expected by the envisaged experiments, considering also a safety margin dealing with the losses in delivering the photon beams to the experimental hutch. A further innovation is a truly coherent photon beam at $0.1-1 \mathrm{MHz}$, that can be obtained with a segmented undulator performing cascades seeded either by the high harmonics of a J-class laser generated in gas or by a EUV FEL oscillator. Another option is represented by a regenerative amplifier, operating with Diamond mirrors at about $4 \AA$. In all cases $10^{10}$ coherent photons are produced.

The techniques presented throughout this work show the capability of the MariX complex to generate a coherent and statistically stable photon beam at X-ray energies, though few difficulties need 
to be considered. The HHG is a widely studied technique, even if the desired energy level limits its use as seeding source to relatively low repetition rates (i.e., less than $100 \mathrm{kHz}$ ). The EUV oscillator-based seeding mechanism is instead compatible with a large repetition rate; high repetition rate oscillators in the EUV /X-rays, however, have not been yet experimentally demonstrated and may give problems related to the behaviour of the long cavity and the technology of the mirrors. The triple cascade presented in both these schemes is moderately beyond the state of the art, for the short period of the last undulator and for the range of radiation wavelengths. The third scheme, based on the regenerative amplifier, relies upon a much simpler undulator structure, but is limited by the technological challenge related to the X-ray optics and transport, and has the disadvantage that cavity, mirrors and optical line are designed for a specific wavelength and is difficultly tunable.

Such sources will fill in the XAS / XMCD (with polarization control from quarterwavelength blades or undulators) and bulk photoemission to become highly efficient probes of matter at the nanoscale but in bulk environments, like buried interfaces of interest in materials science, in-vivo biological samples or catalysers at work. The novel source will therefore create absolutely novel conditions for experiments that cannot be performed satisfactorily at the present and foreseen sources based on storage rings or SASE-FELs. The anticipated performances of MariX Free Electron Laser are well beyond the state of the art of presently FELs in operation, and in the trailing edge of EuXFEL and of the US future superconducting FEL project of reference (LCLS-II).

A new generation accelerator complex is at the core of the MariX facility, dedicated and optimized to ultrafast coherent-X-ray spectroscopy and inelastic photon scattering, and to highly penetrating X-ray imaging of mesoscopic and macroscopic samples. Such facilities will be intrinsically multi-user and multidisciplinary as of the research performed and science output.

Author Contributions: Conceptualization V.P., A.B., A.R.R., L.S. and G.R.; Definition of the scientific case G.G., E.P., L.S. and G.R.; Methodology V.P., M.O.; Methodology of the HHG part A.P; Methodology of the regenerative amplifier part A.T.; Software and data curation M.O., I.D., M.R.C. and S.S.; Writing-original draft preparation, writing-review and editing V.P., M.O.; Supervision V.P., L.S. and G.R.; Funding acquisition L.S.

Funding: This research received no external funding.

Conflicts of Interest: The authors declare no conflict of interest.

\section{References}

1. Faatz, B.; Schreiber, S. First Lasing of FLASH2 at DESY. Synchrotron Radiat. News 2014, 27, 37. [CrossRef]

2. Emma, P.; Akre, R.; Arthur, J.; Bionta, R.; Bostedt, C.; Bozek, J.; Brachmann, A.; Bucksbaum, P.; Coffee, R.; Decker, F.-J.; et al. First lasing and operation of an Angstrom-wavelength free-electron laser. Nat. Photonics 2010, 4, 641-647. [CrossRef]

3. Pile, D. First light from SACLA. Nat. Photonics 2011, 5, 456-457. [CrossRef]

4. Weise, H.; Decking, W. Commissioning and first kasing of the European XFEL. In Proceedings of the 38th International Free-Electron Laser Conference, Santa Fe, NM, USA, 20-25 August 2017.

5. Milne, C.J. SwissFEL: The swiss X-ray free electron laser. Appl. Sci. 2017, 7, 720. [CrossRef]

6. Ko, I.S.; Kang, H.; Heo, H.; Kim, C.; Kim, G.; Min, C.; Yang, H.; Baek, S.Y.; Choi, H.; Mun, G.; et al. Construction and commissioning of PAL-XFEL facility. Appl. Sci. 2017, 7, 479. [CrossRef]

7. Kang, H.S. Hard X-ray FEL Lasing Through BBA and Radiation Spectrum Analysis. In Proceedings of the International Particle Accelerator Conference (IPAC'17), Copenhagen, Denmark, 14-19 May 2017.

8. Wang, D. Soft X-Ray Free-Electron Laser at SINAP. In Proceedings of the Seventh International Particle Accelerator Conference, Busan, Korea, 8-13 May 2016.

9. Raubenheimer, T. LCLS-II-HE FEL Facility Overview. Available online: https:/ / portal.slac.stanford.edu/ sites / conf_public/lclsiihe2016/Documents/160926\%20LCLS-II-HE\%20Raubenheimer.pdf (accessed on 6 July 2019).

10. Marie Facility. Available online: https://www.lanl.gov/science-innovation/science-facilities/marie/index. php (accessed on 6 July 2019). 
11. Elettra Sincrotrone Trieste. Available online: https://www.elettra.trieste.it/lightsources/elettra (accessed on 6 July 2019).

12. Allaria, E.; Appio, R.; Badano, L.; Barletta, W.A.; Bassanese, S.; Biedron, S.G.; Borga, A.; Busetto, E.; Castronovo, D.; Cinquegrana, P.; et al. Highly coherent and stable pulses from the FERMI seeded free-electron laser in the extreme ultraviolet. Nat. Photonics 2012, 6, 699-704. [CrossRef]

13. Rosenzweig, J.; Alesini, D.; Andonian, G.; Boscolo, M.; Dunning, M.; Faillace, L.; Ferrario, M.; Fukusawa, A.; Giannessi, L.; Hemsing, E.; et al. Generation of ultra-short, high brightness electron beams for single-spike SASE FEL operation. Nucl. Instrum. Methods Phys. Res. A 2008, 593, 39-44. [CrossRef]

14. Geloni, G.; Kocharyan, V.; Saldin, E. A novel self-seeding scheme for hard X-ray FELs. J. Mod. Opt. 2011, 58, 1391-1403. [CrossRef]

15. Giannessi, L.; Artioli, M.; Bellaveglia, M.; Briquez, F.; Chiadroni, E.; Cianchi, A.; Couprie, M.E.; Dattoli, G.; Di Palma, E.; Di Pirro, G.; et al. High-order-harmonic generation and superradiance in a seeded free-electron laser. Phys. Rev. Lett. 2012, 108, 164801. [CrossRef]

16. Giannessi, L.; Bellaveglia, M.; Chiadroni, E.; Cianchi, A.; Couprie, M.E.; Del Franco, M.; Di Pirro, G.; Ferrario, M.; Gatti, G.; Labat, M.; et al. Superradiant Cascade in a Seeded Free-Electron Laser. Phys. Rev. Lett. 2013, 110, 044801. [CrossRef]

17. Xiang, D.; Stupakov, G. Echo-enabled harmonic generation free electron laser. Phys. Rev. Spec. Accel. Beams 2009, 12, 030702. [CrossRef]

18. Hemsing, E.; Dunning, M.; Garcia, B.; Hast, C.; Raubenheimer, T.; Stupakov, G.; Xiang, D. Echo-enabled harmonics up to the 75th order from precisely tailored electron beams. Nat. Photonics 2016, 10, 512-515. [CrossRef]

19. Feng, C.; Deng, H.; Zhang, M.; Wang, X.; Chen, S.; Liu, T.; Zhou, K.; Gu, D.; Wang, Z.; Jiang, Z.; et al. Coherent extreme ultraviolet free-electron laser with echo-enabled harmonic generation. Phys. Rev. Accel. Beams 2009, 22, 050703. [CrossRef]

20. Ribič, P.R.; Abrami, A.; Badano, L.; Bossi, M.; Braun, H.; Bruchon, N.; Capotondi, F.; Castronovo, D.; Cautero, M.; Cinquegrana, P.; et al. Coherent soft X-ray pulses from an echo-enabled harmonic generation free-electron laser nature research. Nat. Photonics 2019, 13, 1-7.

21. Dattoli, G.; Palma, E.D.; Petralia, A. Free electron laser oscillator efficiency. Opt. Commun. 2018, 425, 29.

22. Kim, K.; Shvyd'ko, Y.; Reiche, S. A proposal for an X-ray free-electron laser oscillator with an energy-recovery linac. Phys. Rev. Lett. 2008, 100, 244802. [CrossRef] [PubMed]

23. Li, A.K.; JYan, i.; Feng, C.; Zhang, M.; Deng, A.H. High brightness fully coherent X-ray amplifier seeded by a free-electron laser oscillator. Phys. Rev. Accel. Beams 2018, 21, 040702 [CrossRef]

24. Serafini, L.; Bacci, A.; Bellandi, A.; Bertucci, M.; Bolognesi, M.; Bosotti, A.; Broggi, F.; Calandrino, R.; Camera, F.; Canella, F.; et al. MariX, an advanced MHz-class repetition rate X-ray source for linear regime time-resolved spectroscopy and photon scattering. Nucl. Instrum. Meth. A. 2019, 930, 167-172. [CrossRef]

25. Mitri, S.D.; Cornacchia, M. Transverse emittance-preserving arc compressor for highbrightness electron beam-based light sources and colliders. EPL Europhys. Lett. 2015, 109, 62002. [CrossRef]

26. Mitri, S.D. Feasibility study of a periodic arc compressor in the presence of coherent synchrotron radiation. Nucl. Instrum. Methods Phys. Res. A 2016, 806, 184. [CrossRef]

27. Placidi, M.; Mitri, S.D.; Pellegrini, C.; Penn, G. Compact FEL-driven inverse compton scattering gamma-ray source. Nucl. Instrum. Methods Phys. Res. A 2017, 855, 55-60. [CrossRef]

28. Bacci, A.; Conti, M.R.; Bosotti, A.; Cialdi, S.; Mitri, S.D.; Drebot, I.; Faillace, L.; Ghiringhelli, G.; Michelato, P.; Monaco, L.; et al. Two-pass two-way acceleration in a Super-Conducting CW linac to drive low jitters X-ray FELs. Phys. Rev. Accel. Beams 2019, submitted.

29. Yu, L.H.; Babzien, M. ; Ben-Zvi, I.; DiMauro, L.F.; Doyuran, A.; Graves, W.; Johnson, E.; Krinsky, S.; Malone, R.; Pogorelsky, I.; et al. High-gain harmonic-generation free-electron laser. Science 2000, 289, 932-934. [CrossRef] [PubMed]

30. Yu, L.H.; DiMauro, L.; Doyuran, A.; Graves, W.S.; Johnson, E.D.; Heese, R.; Krinsky, S.; Loos, H.; Murphy, J.B.; Rakowsky, G.; et al. First ultraviolet high-gain harmonic-generation free-electron laser. Phys. Rev. Lett. 2003, 91, 074801. [CrossRef]

31. Takahashi, E.J.; Nabekawa, Y.; Mashiko, H.; Hasegawa, H.; Suda, A.; Midorikawa, K. Generation of strong optical field in soft X-ray region by using high-order harmonics. IEEE J. Sel. Top. Quantum Electron. 2004, 10, 1315-1328. [CrossRef] 
32. Lambert, G.; Gautier, J.; Hauri, C.P.; Zeitoun, P.; Valentin, C.; Marchenko, T.; Tissier, F.; Goddet, J.P.; Ribière, M.; Rey, G.; et al. An optimized kHz two-colour high harmonic source for seeding free-electron lasers and plasma-based soft x-ray lasers. New J. Phys. 2009, 11, 083033. [CrossRef]

33. Labat, M.; Bellaveglia, M.; Bougeard, M.; Carré, B.; Ciocci, F.; Chiadroni, E.; Cianchi, A.; Couprie, M.E.; Cultrera, L.; Del Franco, M.; et al. High-gain harmonic-generation free-electron laser seeded by harmonics generated in gas. Phys. Rev. Lett. 2011, 107, 224801. [CrossRef] [PubMed]

34. Ben-Zvi, I.; Yang, K.M.; Yu, L.H. The fresh-bunch technique in FELS. Nucl. Instrum. Methods Phys. Res. A 1992, 318, 726-729. [CrossRef]

35. Reiche, S. GENESIS 1.3: A fully 3D time-dependent FEL simulation code. Nucl. Instrum. Methods Phys. Res. A 1999, 429, 243-248. [CrossRef]

36. Schraeder, S.; Feigl, T.; Duparr, A.; Tannermann, A. EUV reflectance and scattering of Mo/Si multilayers on differently polished substrates. Opt. Express 2007, 15, 13997-14012. [CrossRef]

(c) 2019 by the authors. Licensee MDPI, Basel, Switzerland. This article is an open access article distributed under the terms and conditions of the Creative Commons Attribution (CC BY) license (http:/ / creativecommons.org/licenses/by/4.0/). 